\title{
PDD Symptoms in ADHD, an Independent Familial Trait?
}

\author{
J. S. Nijmeijer • P. J. Hoekstra • R. B. Minderaa • \\ J. K. Buitelaar • M. E. Altink • C. J. M. Buschgens • \\ E. A. Fliers • N. N. J. Rommelse • J. A. Sergeant • \\ C. A. Hartman
}

Published online: 3 December 2008

(C) The Author(s) 2008. This article is published with open access at Springerlink.com

\begin{abstract}
The aims of this study were to investigate whether subtle PDD symptoms in the context of ADHD are transmitted in families independent of ADHD, and whether PDD symptom familiality is influenced by gender and age. The sample consisted of 256 sibling pairs with at least one child with ADHD and 147 healthy controls, aged 5-19 years. Children who fulfilled criteria for autistic disorder were excluded. The Children's Social Behavior Questionnaire (CSBQ) was used to assess PDD symptoms. Probands, siblings, and controls were compared using analyses of variance. Sibling correlations were calculated for CSBQ scores after controlling for IQ, ADHD, and
\end{abstract}

J. S. Nijmeijer • P. J. Hoekstra • R. B. Minderaa • C. A. Hartman Department of Psychiatry, University of Groningen,

University Medical Center Groningen,

Groningen, The Netherlands

J. K. Buitelaar · M. E. Altink · C. J. M. Buschgens · E. A. Fliers · N. N. J. Rommelse

Department of Psychiatry, Radboud University Nijmegen Medical Center, and Karakter, Child and Adolescent Psychiatry University Center Nijmegen,

Nijmegen, The Netherlands

E. A. Fliers

Youth Department, BAVO-Europoort,

Rotterdam, The Netherlands

N. N. J. Rommelse $\cdot$ J. A. Sergeant

Department of Clinical Neuropsychology,

VU University Amsterdam,

Amsterdam, The Netherlands

J. S. Nijmeijer $(\square)$

Department of Child and Adolescent Psychiatry,

University of Groningen,

Hanzeplein 1, PO box 660, 9700 AR Groningen, Netherlands

e-mail: j.nijmeijer@accare.nl comorbid anxiety. In addition, we calculated cross-sibling cross-trait correlations. Both children with ADHD and their siblings had higher PDD levels than healthy controls. The sibling correlation was 0.28 for the CSBQ total scale, with the CSBQ stereotyped behavior subscale showing the strongest sibling correlation $(r=0.35)$. Sibling correlations remained similar in strength after controlling for IQ and ADHD, and were not confounded by comorbid anxiety. Sibling correlations were higher in female than in male probands. The social subscale showed stronger sibling correlations in elder than in younger sibling pairs. Crosssibling cross-trait correlations for PDD and ADHD were weak and not-significant. The results confirm that children with ADHD have high levels of PDD symptoms, and further suggest that the familiality of subtle PDD symptoms in the context of ADHD is largely independent from ADHD familiality.

Keywords ADHD · Autism · PDD - Comorbidity · Children's social behavior questionnaire

\section{Introduction}

Apart from the defining symptoms of inattention, hyperactivity, and/or impulsivity, many children with attention deficit/hyperactivity disorder (ADHD) show problems in social functioning. Although children with ADHD generally do not lack interest in other people, they often fail to properly attune their behavior to other persons and to constantly changing social environments. The key characteristic of the social behavior of a substantial number of children with ADHD can be described as an apparent lack of a full comprehension of the consequences of their behaviors to others (Nijmeijer et al. 2008). This character- 
istic resembles the lack of reciprocity that is one of the defining attributes of the social behavior of children with pervasive developmental disorders (PDD). The similarities in social difficulties suggest a certain degree of overlap between the two disorders, despite their strict segregation in both the Diagnostic and Statistical Manual of Mental Disorders IV-text revision (DSM-IV-TR; American Psychiatric Association 1994) and the International Statistical Classification of Diseases (ICD-10; World Health Organisation 1992). This potential overlap is supported by findings of the presence of symptoms of PDD in children with ADHD, and vice versa. The PDD symptoms that are reported to occur most frequently and in a majority of children with ADHD are impairments in social interaction, especially the inability to conceive other individuals' feelings and thoughts (Buitelaar et al. 1999, Clark et al. 1999, Santosh and Mijovic 2004). Symptoms of communication problems, and stereotyped and repetitive behaviors, have been reported to occur frequently as well in children with ADHD (Clark et al. 1999, Santosh and Mijovic 2004). A striking finding is that children with ADHD have higher scores on measures of PDD not only in comparison to healthy control children, but also compared to children with psychiatric disorders other than ADHD (Buitelaar et al. 1999, Santosh and Mijovic 2004). Especially children with the DSM-IV combined subtype have been recently shown to have elevated levels of PDD-symptoms, with nearly one third of boys and threefourth of girls with combined type ADHD meeting clinical cut-offs for autistic symptomatology (Reiersen et al. 2007).

It has been well established that both ADHD and PDD have a strong genetic background (for reviews see Faraone et al. 2005, Freitag 2007). Interestingly, two studies in community twin samples have reported that variation in ADHD behaviors partially explain variation in PDD behaviors (Constantino et al. 2003, Ronald et al. 2008). In the most recent of the two, Ronald et al. (2008) found genetic influences on autistic and ADHD behaviors in a community twin sample to be overlapping, with PDD and ADHD traits showing a genetic correlation of around 0.50 . These studies add to two other findings indicating that PDD and ADHD originate from similar underlying deficits. First, apart from symptom overlap, overlap in Theory of Mind and executive function deficits between the two disorders has been identified (Buitelaar et al. 1999, Geurts et al. 2004, Happé et al. 2006, Jonsdottir et al. 2006, Nyden et al. 1999, Ozonoff and Jensen 1999, Yuill and Lyon 2007). This may point to a shared underlying endophenotype between ADHD and PDD. Second, genetic linkage findings have partially suggested the same genetic regions of interest between the two disorders (Bakker et al. 2003, Ogdie et al. 2003, Smalley et al. 2002, Yamagata et al. 2002).

Interestingly, the three PDD symptom domains have been suggested to originate from distinct genetic influences in both general population samples (Ronald et al. 2006a, Ronald et al. 2005, Ronald et al. 2006b) and clinical samples (Georgiades et al. 2007, Kolevzon et al. 2004), although the opposite has been found as well (Dyck et al. 2006, Goin-Kochel et al. 2008). Thus far, the potential differential heritablility of the PDD dimensions in the context of ADHD has not been investigated.

Furthermore, in studies regarding the overlap between PDD and ADHD gender has rarely been taken into account. Given the higher prevalence of PDD in boys, one would expect lower levels of PDD in girls with ADHD. This was indeed what was found for autistic symptoms in the international IMAGE sample (Mulligan et al. 2008). However, clinically relevant PDD symptomatology in children with ADHD has also been found to be more prevalent in girls than in boys (Reiersen et al. 2007).

Another understudied topic is how age influences PDD symptoms in children with ADHD. Although social problems are associated with ADHD from early childhood through adolescence and young adulthood (Nijmeijer et al. 2008) this has not yet been investigated for PDD symptoms as such.

In the present study, these issues have been addressed using phenotypic data of Dutch children with ADHD and one of their siblings, which were collected as part of the IMAGE project. Children with autism were excluded from participation, which resulted in a sample where all variation in PDD severity except for the most severe end was represented. Most presently available PDD screening instruments are insufficient for assessing milder, more subtle PDD symptoms, as they are designed to screen for severe forms of PDD. Therefore, in the Dutch IMAGE sample the Children's Social Behavior Questionnaire (Hartman et al. 2006) was used. The CSBQ is particularly suited to measure the continuous distribution of PDD traits in the population as well as in clinical groups other than PDD (Hartman et al. 2006, Luteijn et al. 2000).

The questions we sought to answer in this study are: Firstly, can we confirm previous findings of elevated levels of PDD symptomatology in children with ADHD? Secondly, can we confirm the familiality of PDD symptoms in children with of ADHD? If so, does subtle PDD symptom familiality depend on ADHD familiality? Thirdly, does this familiality differ for different PDD dimensions? And fourthly, are similar results found for boys and girls with ADHD, respectively, and for younger and elder sibling pairs?

\section{Methods}

Subjects

This study reports on data from Dutch participants of IMAGE. IMAGE is a collaborative study in eight European 
countries (Belgium, Germany, Ireland, Israel, Spain, Switzerland, the Netherlands, and the United Kingdom) that aims to identify genes that increase the risk of ADHD using linkage and association strategies. All probands in IMAGE were aged 5 to 17 at the time of entry into the study, and are of European Caucasian descent. Entry criteria for the probands were a clinical diagnosis of DSM-IV combined subtype of ADHD and having one or more full siblings aged 5-19 available for ascertainment of clinical information and DNA collection. Exclusion criteria applying to both probands and siblings included an IQ $<70$, autism (see next section for a detailed description of assessment), epilepsy, brain disorders, and any genetic or medical disorder associated with externalizing behaviors that might mimic ADHD. Whenever possible, families withdrew stimulant medication for 1 week prior to research assessment to allow for more accurate ascertainment of information on recent ADHD symptom characteristics and severity. For this study, probands with at least one sibling (regardless of his/her ADHD-status) were recruited from families referred to several participating academic child psychiatric outpatient clinics, or from the members of the Dutch Parents' Association. They had undergone clinical evaluations by a pediatrician or child psychiatrist in the recent past. CSBQ data were available for 256 families. For this study, only one sibling was selected at random in case families with two or more participating siblings.
Additional control families were recruited from main stream primary and high-schools from the same geographical regions as the participating families with an ADHD proband. Principals were asked permission to contact the parents of their pupils. Parents who gave permission received questionnaires by mail. Control children had to obtain non-clinical scores (i.e., $<63$ ) on both the Conners ${ }^{6}$ parent and the Conners' teacher ADHD/DSM-IV totalsubscale. The control sample consisted of sibpairs and singletons, aged 5 to 19 . From each pair, one sibling was selected with preference given to boys over girls.

The final sample was formed by 256 DSM-IV ADHD combined type probands (219 boys) and 256 siblings (131 boys), and 147 control children (89 boys). Characteristics of these groups can be found in Table 1 .

\section{Diagnostic Instruments}

Children already diagnosed with ADHD as well as their siblings were screened using the standard IMAGE procedures as described by Brookes et al. (2006). Briefly, screening questionnaires, i.e., the parent and teacher Conners' rating scales, long forms (Conners 1996), and the parent and teacher Strength and Difficulties Questionnaires (Goodman 1997), were used to identify children with ADHD-symptoms. T-scores $\geq 63$ on any Conners' ADHD/ DSM-IV subscale and scores $>90$ th percentile on the parent

Table 1 Characteristics for probands, siblings, and controls

\begin{tabular}{|c|c|c|c|c|c|c|}
\hline & Probands $(n=256)$ & Siblings $(n=256)$ & Controls $(n=147)$ & & & \\
\hline Characteristic & Mean (SD) & Mean (SD) & Mean (SD) & ANOVA's & $\eta_{P}^{2}$ & $\mathrm{p}$ \\
\hline Age & $11.3(2.6)$ & $10.9(3.5)$ & $11.2(3.0)$ & $\mathrm{F}(2,650)=1.03$ & & 0.36 \\
\hline IQ & $98.3(12.5)$ & $102.0(11.6)$ & $106.6(9.9)$ & $\mathrm{F}(2,646)=23.04$ & 0.07 & $<0.001^{\mathrm{a}}$ \\
\hline \multicolumn{7}{|l|}{ ADHD-symptom-scores } \\
\hline Conners' DSM-IV total & $76.5(8.7)$ & $55.3(12.3)$ & $46.5(4.4)$ & $F(2,656)=539.82$ & 0.62 & $<0.001^{\mathrm{b}}$ \\
\hline DSM-IV inattentive & $70.7(8.4)$ & $54.1(11.8)$ & $46.4(5.0)$ & $\mathrm{F}(2,656)=367.88$ & 0.53 & $<0.001^{\mathrm{b}}$ \\
\hline DSM-IV hyperactive-impulsive & $78.7(9.1)$ & $55.6(12.8)$ & $47.3(4.8)$ & $\mathrm{F}(2,656)=555.36$ & 0.63 & $<0.001^{\mathrm{b}}$ \\
\hline \multicolumn{7}{|l|}{ PDD-symptoms } \\
\hline CSBQ-total & $72.0(13.1)$ & $57.5(12.8)$ & $46.4(6.2)$ & $F(2,656)=257.05$ & 0.44 & $<0.001^{\mathrm{b}}$ \\
\hline Tuned & $92.8(12.6)$ & $58.1(12.5)$ & $46.6(7.4)$ & $\mathrm{F}(2,656)=252.77$ & 0.44 & $<0.001^{\mathrm{b}}$ \\
\hline Social & $62.1(15.5)$ & $54.5(14.3)$ & $47.5(6.5)$ & $F(2,656)=56.95$ & 0.15 & $<0.001^{\mathrm{b}}$ \\
\hline Orientation & $73.4(15.4)$ & $55.7(13.3)$ & $46.3(5.8)$ & $\mathrm{F}(2,656)=230.90$ & 0.41 & $<0.001^{\mathrm{b}}$ \\
\hline Understanding & $69.4(13.7)$ & $57.8(13.4)$ & $48.0(7.4)$ & $F(2,656)=145.3$ & 0.31 & $<0.001^{\mathrm{b}}$ \\
\hline Stereotyped & $68.4(18.9)$ & $53.1(13.6)$ & $47.8(7.7)$ & $\mathrm{F}(2,656)=109.15$ & 0.25 & $<.001^{\mathrm{b}}$ \\
\hline Change & $61.7(16.1)$ & $52.5(11.5)$ & $48.8(9.3)$ & $F(2,656)=54.43$ & 0.14 & $<0.001^{\mathrm{c}}$ \\
\hline Gender & N (\%) & N (\%) & N (\%) & Kruskal-Wallis & & \\
\hline Boy & $219(85.5)$ & $131(51.2)$ & $89(60.5)$ & $\mathrm{H}(2)=71.04$ & & $<0.001^{\mathrm{d}}$ \\
\hline Girl & $37(14.5)$ & $125(49.8)$ & $58(39.5)$ & & & \\
\hline
\end{tabular}

$A D H D$ attention-deficit/hyperactivity disorder, $A N O V A$ analysis of variance, $C S B Q$ child social behavior questionnaire, $D S M-I V$ diagnostic and statistical manual of mental disorders, 4 th edition, $P D D$ pervasive developmental disorders

${ }^{a}$ probands $<$ siblings $<$ controls $(p<0.001)$

${ }^{\mathrm{b}}$ probands $>$ siblings $>$ controls $(p<0.001)$

${ }^{c}$ probands $>$ siblings $>$ controls $(p<0.01)$

$\mathrm{d}$ probands $>$ siblings $(p<.001)$, siblings $=$ controls 
or teacher SDQ-hyperactivity scale were considered as clinical. Concerning all patients already diagnosed with ADHD and siblings scoring clinically on the parent or teacher Conners' or SDQ, a semi-structured, standardized, investigator-based interview was administered separately for each child; the Parental Account of Childhood Symptoms (PACS; Taylor 1986). The PACS covers DSM-IV symptoms of ADHD, conduct disorder, oppositional defiant disorder, anxiety, mood, and other internalizing disorders. The most prevalent comorbid disorders in probands as assessed by the PACS were oppositional defiant disorder (152 probands, $59.3 \%$ ) and anxiety disorder (147 probands, $57.4 \%$ ). 45 probands (17.6\%) had no comorbid disorder.

A standardized algorithm was applied to the PACS to derive each of the 18 DSM-IV ADHD-items, providing operational definitions for each behavioral symptom. These were combined with items that scored 2 or 3 on the ADHD/ DSM-IV total-subscale from the Conners' Teacher Rating Scale-Revised: Long Form (Conners' teacher), to generate the total number of items from the DSM-IV symptom checklist. Situational pervasiveness was defined as some symptoms occurring within two or more different situations from the PACS interview, as well as the presence of one or more symptoms scoring two or more from the ADHD/ DSM-IV total subscale of the teacher rated Conners'.

Children were excluded from participation in IMAGE when classical or atypical autism was diagnosed. This was regarded to be present in case of a clinical score (i.e., $\geq 15$ ) on the SCQ and the presence of PDD symptoms in at least two out of three DSM-IV autism domains, together with a developmental delay in at least one autism domain before the age of 3 , as assessed by the section on autistic disorder of the PACS interview. Weight was given especially to symptoms that distinguish PDD from ADHD, and a lack of relationships with peers alone was not sufficient for social impairment of the autistic type. The mean SCQ scores in the final sample were 9.2 (sd 5.6) for probands and 5.4 (sd 4.4) for siblings. No SCQ data were available for controls. Reported SCQ scores in normal controls range from 4.2 (Rutter et al. 1999) to 1.69 (Farzin et al. 2006).

Measures

\section{PDD Symptoms}

The CSBQ contains 49 items scored on a 3-point Likertscale. It contains items that refer directly to DSM-IV criteria for autism, but it also represents less severe variations of these criteria as well as PDD associated problems, such as problems in executive functioning and disruptive behavior. Because of this it also captures behavior problems as seen in children with milder variants of PDD. Age and gender specific T-scores were used for all analyses in this paper, and were calculated based on a general population sample of 2,507 Dutch children aged 5 to 17 who have been described in the Dutch CSBQ manual (Hartman et al. 2007a, 2007b). CSBQ items are grouped in six subscales called "not optimally tuned to the social situation" (tuned; 11 items addressing emotional overreacting and stubbornness/disobedience), "reduced contact and social interest" (social; 12 items), "orientation problems in time, place, or activity" (orientation; 8 items), "difficulties in understanding of social information" (understanding; 7 items), "fear of and resistance to changes" (change; 3 items), and "stereotyped behavior" (stereotyped; 8 items). Estimates for internal, test-retest, and inter-rater reliability, and for convergent and divergent validity were good (Hartman et al. 2006). The CSBQ appears to differentiate between autism and PDDNOS on the one hand, and PDDNOS and ADHD on the other (Geurts et al. 2008, Hartman et al. 2006). Furthermore, to assess the content validity of the CSBQ, it has previously been compared to an autism screening instrument, the Autism Behavior Checklist (ABC; Krug 1980). A strong correlation of 0.75 was found between the total scores of both questionnaires in a large Dutch population sample (Luteijn et al. 2000) confirming that what the CSBQ measures correlates strongly with what more stringent autism measures pick up. In the present sample, the Pearson's correlation between the SCQ and CSBQ (raw) total score was $0.52(p<.01)$, indicating that these questionnaires assess similar but not identical behaviors.

Two CSBQ subscales, i.e., the tuned and the orientation subscale, tap dysfunctional social behaviors that are conceptually closely related to the ADHD dimensions hyperactivity/ impulsivity and attention problems, respectively. These behaviors occur frequently in children with PDD, as is recognized in DSM-IV as well. By including these subscales in the analyses it will be possible to investigate whether the relation between ADHD and PDD symptoms is carried mainly by these behaviors, or also by more PDD specific behaviors represented in the other subscales.

\section{ADHD-symptoms}

The Conners' parent rating scale, long form was applied as a measure of ADHD symptom severity. Items are scored on a four-point Likert scale. Analyses were carried out using T-scores for the DSM-IV symptoms subscales, i.e., the inattentive, the hyperactive-impulsive and the total scale. The latter is calculated by adding up the inattentive and hyperactive-impulsive subscales. We used the DSM-IV scales because these most closely reflect the current ADHD definition, whereas other scales contain some items that are ADHD-related, but somewhat less ADHD specific. For the 
9 siblings who were 18 or 19 at the time of data-collection we applied the 15-17 year age norms.

\section{Anxiety}

The anxious-shy scale of the Conners' parent rating scale, long form, was used to assess anxiety, which is a potential confounding factor of the association between ADHD and PDD. T-scores were used for this scale as well, which consists of 6 items scored on a four-point Likert scale.

\section{Intelligence}

Full-scale IQ was estimated by four subtests of the Wechsler Intelligence Scale for Children - Third Version (WISC-III) or Wechsler adult intelligence scaleThird version (WAIS-III) depending on the child's age: Vocabulary, Similarities, Block Design, and Picture Completion (Wechsler 2000, Wechsler 2002). The subtests are known to correlate between $0.90-0.95$ with fullscale IQ (Groth-Marnat 1997). As the WISC-III is not officially suited for 5 year-olds, data from these children (1 proband and 9 siblings) were excluded from the analyses where IQ was included as a covariate.

\section{Analyses}

Group differences in age, IQ, and T-scores on the CSBQ and the Conners' parent DSM-IV symptom subscales between probands, siblings, and controls were assessed with univariate analyses of variance (ANOVAs). Effect sizes $\eta^{2} 0.01,0.06$ and 0.14 were considered small, medium and large, respectively, following Cohen's standard (1988). To test whether subtle PDD symptoms occur more frequently in children with ADHD and their siblings than in controls, the following contrasts were defined: probands versus siblings, and siblings versus controls. The KruskalWallis test, followed by Mann-Whitney tests, was used to determine differences in gender distribution between probands, siblings and controls.

The relation between ADHD severity and PDD severity was assessed by calculating Pearson's correlations between Conners' parent total scores and CSBQ subscale scores in probands (phenotypic correlations). Correlations were also calculated between the CSBQ subscales and the inattentive and hyperactive-impulsive Conners' parent subscales, respectively, to investigate whether the relation between ADHD and PDD symptoms was driven by either ADHD component.

The familiality of PDD symptoms was estimated by calculating Pearson's correlations between proband and sibling scores (sibling correlations) on the CSBQ scales. To investigate whether findings were robust these analyses were repeated after partialling out variance based on differences in IQ between siblings. For these analyses IQ was included as a covariate in a model in which the subjects' CSBQ subscale score was the dependent variable. The residuals of this analysis were used to calculate sibling correlations.

To further evaluate the familiality of PDD symptoms, we expanded these analyses by adding the Conners' parent DSM-IV-total score to the covariates. Correlations between siblings were calculated for the residuals of this model. From these analyses, there are two possible outcomes. First, if including the covariate does not eliminate or diminish the sibling correlations, then the covariate does not uniquely account for the familiality of the other measure. Second, if including the covariate diminishes the sibling correlation, a common genetic mechanism could be shared by both measures. A similar approach can be found in articles by Szatmari et al. (2008) and Raskind et al. (2000). These analyses were repeated with the Conners' parent DSM-IV inattentive and hyperactive-impulsive scale instead of the total scale. Furthermore, sibling correlations were calculated for the residuals of a model in which the Conners' parent anxiousshy scale was included as a covariate in addition to IQ and the Conners' parent DSM-IV-total score. This was done to verify whether anxiety confounds the association between ADHD and PDD symptom familiality.

The relation between ADHD and PDD symptom familiality was further tested by calculating cross-sibling cross-trait correlations (cross-correlations). If ADHD and PDD share familial influences, sibling Conners' parent DSM-IV symptom scores should be correlated with proband CSBQ scores.

Sibling correlations were calculated separately for male and female probands, and for younger and elder sibling pairs, respectively. For the latter, a median-split based on proband age was made. Siblings were divided in younger siblings ( $<11$ years) and elder siblings ( $\geq 11$ years), based on the proband age median. Age concordant pairs (i.e., both siblings and proband either under or above the median split) were formed, with 88 younger and 65 elder sibling pairs. To determine whether sibling pairs with male and female probands, and younger and elder sibling pairs, respectively, showed different correlations, Fisher's z-score transformations and t-tests were calculated using freely available software (Preacher 2002).

All other analyses were carried out using Statistical Package for the Social Sciences Version 15. We used an alpha of 0.01 to indicate statistical significance for all analyses on the full sample, and an alpha of 0.05 for the analyses of male and female probands and younger and elder sibling pairs, respectively. 


\section{Results}

Table 1 presents general characteristics and symptom scores of probands, siblings, and controls. ADHD probands had the lowest IQ, followed by their siblings. Controls had the highest IQ. The percentage of males was higher in the proband group than in the sibling and control group, which in turn had similar male to female distributions. All Tscores on the CSBQ scales and the Conners' parent DSMIV-symptoms scales showed significant group differences, with large effect sizes for all scales. Planned contrasts indicated that probands had more subtle PDD and ADHD symptoms than siblings, and that siblings had more PDD and ADHD symptoms than controls.

Phenotypic correlations between the PDD and ADHD symptom scores are presented in Table 2. Correlations between CSBQ subscales and the Conners' parent DSMIV-symptom scores were all statistically significant, with the exception of that between the CSBQ social and the Conners'parent DSM-IV hyperactive impulsive scale. The CSBQ total score correlated $0.40(p<0.001)$ with the Conners' parent DSM-IV-total score. As expected, the tuned and the orientation subscales correlated the strongest with the Conners' parent DSM-IV-total score, namely 0.38 ( $p$ $<0.001)$ and $0.42(p<0.001)$, respectively. CSBQ subscales that measure core problems of PDD, i.e., social, understanding, stereotyped and change, showed somewhat lower correlations with the Conners' parent DSM-IV total score (between 0.16 and 0.27 ). These positive correlations confirm the association of PDD with ADHD symptoms. Correlations between the CSBQ and the Conners' parent DSM-IV inattentive and hyperactive-impulsive scales, respectively, showed no apparent differences in strength for all but the social and orientation subscales (see Table 2). These appeared to correlate stronger with the Conners' parent inattentive than with the hyperactive-impulsive subscale ( 0.23 and 0.46 for the inattentive, and 0.08 and 0.27 for the hyperactive-impulsive scale, respectively).

Table 3 shows sibling correlations for the CSBQ subscales. Considering the sibling correlations for the Tscores (second column), evidence of familiality was found. All but two of the PDD subscales showed significant correlations, with the CSBQ total symptom scores corre- lating $0.28(\mathrm{p}<0.001)$. The CSBQ subscale that showed the strongest sibling correlation was the stereotyped subscale, $r=0.35, \mathrm{p}<0.001$.

Subsequently, sibling correlations for the CSBQ subscale scores were calculated correcting for IQ. Results can be found in the third column of Table 3. Inclusion of IQ in the analyses did not appear to change the strength of the crosscorrelations. Furthermore, cross-correlations of the CSBQ total score, and the subscales orientation and stereotyped remained significant after correcting for the Conners' parent DSM-IV-total score. See Table 3, fourth column for the exact strength of the correlations. The results of the familiality analyses were similar when the Conners'parent inattentive or hyperactive-impulsive subscale were used instead of the total scale (see Table 3, fifth and sixth columns). Anxious-shy behavior did not appear to be a confounding factor in the familiality of PDD symptoms in children with ADHD, as can be seen in the last column of Table 3.

The independence of the ADHD and PDD symptom familiality was also suggested by the cross-correlations between proband PDD symptoms and sibling ADHD scores, which were all very weak and non-significant (see Table 4).

In Table 5 characteristics of the probands used for the subgroup analyses based on gender and age can be found. Female probands showed significantly higher T-scores than male probands for almost all ADHD and PDD subscales. The exception was the CSBQ tuned subscale, which did not differ significantly between boys and girls. Small effect sizes were found for all differences in CSBQ subscale scores. A large effect size was found for the Conners' DSM-IV total scale, and a medium effect size for the hyperactive-impulsive scale. Differences between scores of probands of younger and elder sibling pairs were significant for the CSBQ tuned, social and orientation subscale (small effect sizes), and the Conners' DSM-IV hyperactiveimpulsive scale (medium effect size), with younger probands scoring higher on the first two scales, and elder on the latter two.

When sibling correlations were calculated for male and female probands separately, male probands generally showed weaker sibling correlations than female probands

Table 2 Within proband correlations between PDD symptoms and ADHD symptoms

\begin{tabular}{|c|c|c|c|c|c|c|c|}
\hline & CSBQ-total & Tuned & Social & Orientation & Understanding & Stereotyped & Change \\
\hline Conners' DSM-IV total & $0.40^{*}$ & $0.38 *$ & $0.16^{* *}$ & $0.42 *$ & $0.27 *$ & $0.24 *$ & $0.26^{*}$ \\
\hline DSM-IV inattentive & $0.40^{*}$ & $0.32 *$ & $0.23 *$ & $0.46^{*}$ & $0.25^{*}$ & $0.25^{*}$ & $0.25^{*}$ \\
\hline DSM-IV hyperactive-impulsive & $0.30^{*}$ & $0.35^{* *}$ & 0.08 & $0.27 *$ & $0.21 *$ & $0.19 *$ & $0.21^{*}$ \\
\hline
\end{tabular}

$A D H D$ attention-deficit/hyperactivity disorder, CSBQ children's social behavior questionnaire, $D S M$ - $I V$ diagnostic and statistical manual of mental disorders, 4 th edition, $P D D$ pervasive developmental disorders

$* p<0.001, * * 0.001<p<0.01$ 
Table 3 Sibling correlations for proband and sibling PDD symptoms

\begin{tabular}{|c|c|c|c|c|c|c|}
\hline & A $(n=256)$ & B $(n=246)$ & $\mathrm{C}(n=246)$ & $\mathrm{D}(n=246)$ & $\mathrm{E}(n=246)$ & $\mathrm{F}(n=246)$ \\
\hline$C S B Q$-total & $0.28 *$ & $0.27 *$ & $0.30^{*}$ & $0.29 *$ & $0.25 *$ & $0.27 *$ \\
\hline Tuned & $0.16^{*}$ & 0.15 & 0.12 & 0.13 & 0.08 & 0.08 \\
\hline Social & $0.18^{*}$ & 0.13 & 0.12 & 0.13 & 0.11 & 0.1 \\
\hline Orientation & $0.19 *$ & 0.17 & $0.26^{*}$ & $0.25^{*}$ & $0.19 * *$ & $0.24 *$ \\
\hline Understanding & 0.15 & 0.15 & 0.13 & 0.1 & 0.14 & 0.13 \\
\hline Stereotyped & $0.35 *$ & $0.36^{*}$ & $0.37 *$ & $0.35^{*}$ & $0.35^{*}$ & $0.36^{*}$ \\
\hline Change & 0.09 & 0.11 & 0.09 & 0.1 & 0.08 & 0.09 \\
\hline
\end{tabular}

$\mathrm{A}=$ Correlations for CSBQ T-scores; $\mathrm{B}=$ correlations for CSBQ T-score residuals, i.e., variance remaining after inclusion of IQ as covariate; $\mathrm{C}=$ correlations as under B, but with Conners' DSM-IV total score added to covariates; D = correlation as under C, but with Conners' DSM-IV inattention score instead of total score; $\mathrm{E}$ = correlation as under $\mathrm{C}$, but with Conners' DSM-IV hyperactive-impulsive score instead of total score; $\mathrm{F}=$ correlation as under $\mathrm{C}$, but with Conners' anxious-shy score added to covariates

$C S B Q$ children's social behavior questionnaire, DSM-IV diagnostic and statistical manual of mental disorders, 4th edition, $P D D$ pervasive developmental disorder

$* p<0.001 ; * * .001<p<0.01$

(Table 6, columns 2 and 3). For the tuned scale, genderdifferences between the correlations for the T-scores, the Conners' parent DSM-IV total corrected residuals, and the residuals corrected for the Conners' parent anxious-shy scale reached statistical significance $(z=2.21, p=0.03 ; z=$ $2.51, p=0.01$, and $z=2.40, p=0.02$, respectively), with female probands showing stronger sibling correlations than male probands.

Results for the age-matched sibling pairs can be found in the right two columns of Table 6. Younger sibling pairs showed weaker correlations on the social scale than did elder pairs, with significant differences for the T-scores and the IQ corrected data $(z=-2.21, p=0.03$ and $z=-2.29, p=$ 0.02 , respectively). Differences in sibling correlations between younger and elder pairs for the other subscales were not significant. For elder sibling papers, correlations appeared to decrease in strength when the Conners' DSMIV total score was included as a covariate, especially for the tuned scale. This suggests that the relation between the familiality of ADHD and PDD symptoms may be age dependent, with possibly more shared familiality between ADHD and PDD symptoms for elder sibling pairs.

\section{Discussion}

The current study confirms the high levels of PDD symptoms in children with ADHD (Clark et al. 1999, Luteijn et al. 2000, Reiersen et al. 2007, Santosh and Mijovic 2004). Our study sample consisted of children with ADHD combined type, a subtype that has previously been reported to have the highest levels of PDD symptoms compared to other ADHD subtypes (Reiersen et al. 2007). Indeed, subtle PDD traits covered by the CSBQ were elevated in the children with ADHD compared to their siblings, with sibling scores still well above those of healthy control children. The elevations in PDD symptoms were not restricted to a specific subset or subscale of PDD symptoms, but covered the whole PDD spectrum, again in line with previous findings (Clark et al. 1999, Reiersen et al. 2007, Santosh and Mijovic 2004).

A positive relation was found between ADHD and PDD symptoms. This endorses the association between ADHD and PDD. However, ADHD-PDD correlations were of modest strength, indicating that PDD symptoms as measured in this study should not be considered a mere proxy for ADHD

Table 4 Cross-correlations between proband PDD symptoms and silbing ADHD symptoms

\begin{tabular}{|c|c|c|c|c|c|c|c|}
\hline & \multicolumn{7}{|c|}{ Proband $(n=256)$} \\
\hline & $C S B Q$-total & Tuned & Social & orientation & understanding & stereotyped & change \\
\hline \multicolumn{8}{|l|}{ Sibling $(n=256)$} \\
\hline Conners' DSM-IV total & 0.06 & 0.10 & 0.06 & 0.01 & 0.04 & 0.02 & 0.03 \\
\hline DSM-IV inattentive & 0.06 & 0.08 & 0.03 & 0.03 & 0.06 & 0.01 & 0.02 \\
\hline DSM-IV hyperactive-impulsive & 0.06 & 0.09 & 0.07 & -0.01 & 0.01 & 0.04 & 0.03 \\
\hline
\end{tabular}

$A D H D$ attention-deficit/hyperactivity disorder, $D S M-I V$ diagnostic and statistical manual of mental disorders, 4th edition, $P D D$ pervasive developmental disorders, $C S B Q$ child social behavior questionnaire 
severity, but point to a different set of problems. As expected beforehand, correlations were the weakest for the most typical PDD subscales, but even the subscales that are conceptually related to ADHD correlated only moderately with the Conners' scores. For most CSBQ subscales, there was no indication that either symptoms of attention deficit or of hyperactivity-impulsivity alone could explain the association. The most outspoken exception was found for the CSBQ social subscale, which showed a weak and non-significant correlation with hyperactive-impulsive symptoms in contrast to symptoms of attention deficits. This suggests that children with combined type ADHD who have mainly inattention problems may show more withdrawn behavior than primarily hyperactive-impulsive children.

Our findings furthermore suggest that PDD symptoms in the context of ADHD are familial, and that this familiality is largely independent from that of ADHD. This finding is strengthened by the fact that IQ and comorbid anxiety were found to be unlikely factors confounding the familiality of PDD symptoms. These results speak against substantial common genetic factors for ADHD and PDD.

This finding is somewhat surprising given that two studies that have previously addressed the familiality of PDD symptoms point to a shared etiology between ADHD and PDD (Constantino et al. 2003, Ronald et al. 2008). It should be noted, however, that both studies involved general population twin samples rather than clinical samples. Interestingly, in a previous study involving the IMAGE sample, but measuring more stringent autism symptoms, the familiality of PDD and ADHD also appeared to be largely independent, as indicated by rather weak PDD-ADHD correlations between siblings (Mulligan et al. 2008). Our IMAGE findings clearly need independent replication, however.

It should be noted that our sibling-pair design allows us to report on familiality rather than heritability. However, previous twin studies suggest PDD is highly heritable (Muhle et al. 2004) and that genetic effects rather than shared environmental factors are largely responsible for sibling similarity in PDD symptomatology. Thus, familiality estimates as identified in the present study are likely to be mostly resulting from genetic factors.

It has been reported that different PDD dimensions may be genetically independent (Georgiades et al. 2007, Kolevzon et al. 2004, Ronald et al. 2005, 2006a, 2006b). In line with this, we found different sibling correlations for the different CSBQ subscales. The CSBQ subscale 'stereotyped behavior' appeared to show the strongest correspondence between siblings. It contains items referring to repetitive movements and sensory stereotypies. The potential dissimilarity in heritability of different PDD dimensions in children with ADHD is a new finding, and needs to be considered in future research. 
Table 6 Sibling correlations for subgroups

Correlations for CSBQ, T-scores

\begin{tabular}{|c|c|c|c|c|}
\hline & $\begin{array}{l}\text { Male } \\
\text { probands } \\
(n=219)\end{array}$ & $\begin{array}{l}\text { Female } \\
\text { probands } \\
(n=37)\end{array}$ & $\begin{array}{l}\text { Younger } \\
\text { sibpairs } \\
(n=88)\end{array}$ & $\begin{array}{l}\text { Elder } \\
\text { sibling } \\
\text { pairs } \\
(n=65)\end{array}$ \\
\hline CSBQ-total & $0.23 * *$ & $0.44 * *$ & $0.35^{* *}$ & $0.33 * *$ \\
\hline Tuned & 0.09 & $0.46^{* *}$ & $0.28 * *$ & $0.26^{*}$ \\
\hline Social & 0.13 & $0.33^{*}$ & 0.03 & $0.38 * *$ \\
\hline Orientation & 0.15 & 0.24 & 0.18 & $0.31 *$ \\
\hline understanding & 0.13 & 0.24 & 0.13 & 0.12 \\
\hline stereotyped & $0.33 * *$ & $0.48^{* *}$ & $0.32 * *$ & $0.37 * *$ \\
\hline change & 0.03 & 0.31 & 0.21 & -0.04 \\
\hline \multicolumn{5}{|c|}{$\begin{array}{l}\text { Correlations for CSBQ T-score residuals (variance remaining } \\
\text { after inclusion IQ as covariate) }\end{array}$} \\
\hline CSBQ-total & $0.24 * *$ & $0.35^{*}$ & $0.37 * *$ & $0.37 *$ \\
\hline tuned & 0.09 & $0.39 *$ & $0.29 * *$ & 0.25 \\
\hline social & 0.07 & 0.24 & 0.03 & $0.39 * *$ \\
\hline orientation & $0.16^{*}$ & 0.09 & $0.23 *$ & $0.33 *$ \\
\hline understanding & $0.15^{*}$ & 0.09 & 0.18 & 0.17 \\
\hline stereotyped & $0.31 * *$ & $0.52 * *$ & $0.43 * *$ & $0.39 * *$ \\
\hline change & 0.05 & 0.29 & $0.22 *$ & 0.06 \\
\hline \multicolumn{5}{|c|}{$\begin{array}{l}\text { Correlations for CSBQ T-score residuals, Conners' DSM-IV total score } \\
\text { added to covariates }\end{array}$} \\
\hline CSBQ-total & $0.25 * *$ & $0.53 * *$ & $0.40 * *$ & 0.20 \\
\hline tuned & 0.06 & $0.48 * *$ & $0.26^{*}$ & 0.05 \\
\hline social & 0.08 & 0.22 & 0.03 & $0.30 *$ \\
\hline orientation & $0.24 * *$ & $0.36^{*}$ & $0.27 *$ & 0.26 \\
\hline understanding & 0.12 & 0.24 & 0.12 & 0.07 \\
\hline stereotyped & $0.30 * *$ & $0.60^{* *}$ & $0.42 * *$ & $0.33 *$ \\
\hline change & 0.05 & 0.25 & $0.23 *$ & -0.07 \\
\hline \multicolumn{5}{|c|}{$\begin{array}{l}\text { Correlations for residuals, Conners' anxious-shy score added to } \\
\text { covariates }\end{array}$} \\
\hline CSBQ-total & $0.22 * *$ & $0.46^{* *}$ & $0.31 * *$ & 0.21 \\
\hline tuned & 0.03 & $0.44 *$ & 0.17 & 0.03 \\
\hline social & 0.07 & 0.15 & -0.04 & $0.29 *$ \\
\hline orientation & $0.23 * *$ & 0.31 & $0.24 *$ & 0.26 \\
\hline understanding & 0.11 & 0.28 & 0.07 & 0.11 \\
\hline stereotyped & $0.30^{* *}$ & $0.55^{* *}$ & $0.38 * *$ & $0.32 *$ \\
\hline change & 0.08 & 0.13 & 0.15 & 0.06 \\
\hline
\end{tabular}

CSBQ children's social behavior questionnaire, DSM-IV diagnostic and statistical manual of mental disorders, 4 th edition

**sibling correlation $p<0.01 ; *$ sibling correlation $p<0.05$

In line with the study by Reiersen et al. (2007), we found elevated levels of subtle PDD symptoms in both boys and girls with ADHD. In fact, girls with ADHD had even higher gender and age specific CSBQ scores than boys, also similar to what Reiersen et al. (2007) found. Therefore, despite the fact that boys are overrepresented in PDD in general, in children affected with combined type ADHD girls are at least as affected with PDD symptoms as are boys. Furthermore, our results suggest that PDD symptoms are more familial when girls are affected, although this needs to be confirmed in a twin sample. This is inconsistent with previous studies indicating that the familial loading is similar in families of boys and girls with PDD, and that that the genes that influence autistic traits are the same for both genders (Constantino and Todd 2003, Goin-Kochel et al. 2007). It should be noted, however, that these studies pertain to PDD while our study focused on subtle PDD symptoms within ADHD.

We observed some differences in familiality for subtle PDD traits in younger versus elder sibling pairs, especially with regard to the social subscale. As far as we know, no data have been published about heritability changes depending on age for PDD symptoms. Nevertheless, it has been found that prosocial behavior shows declining shared environmental and increasing genetic influences with increasing age (Knafo and Plomin 2006, Scourfield et al. 2004). This corresponds with our findings of higher familiality of the social scale, which is negatively correlated with prosocial behavior, in elder sibling pairs.

One of the limitations of this study is that it involved clinically referred children with combined type ADHD. Our findings in this highly selected sample therefore cannot be translated directly to ADHD in the general population. Furthermore, despite a diagnosis of autism being one of the exclusion criteria of the IMAGE study, a few children with comorbid PDD may still have been included in our sample. We think that as such our sample is representative of current clinical practice, where sometimes PDD may go unnoticed when ADHD symptoms dominate the clinical picture. Another issue is that, perhaps besides a rare exception, the majority of children in our sample had PDD traits below the autism cut-off. We do not know whether the underlying genetic factors for these subtle PDD features are the same for full blown autistic symptoms. However, findings from a study using the social responsiveness scale, an instrument similar to the CSBQ, do suggest so (Constantino et al. 2006). Although representative of clinically relevant ADHD, our sample was predominantly male. We found interesting gender differences with regard to the familiality of PDD symptoms, but these findings need replication in samples with more females represented. Also, the cross-sectional nature of our study limits the interpretation of the age effects, which clearly deserve further study in a longitudinal design. Finally, we used parent reports only whereas, ideally, a detailed developmental history and observational data should be collected to assess autistic symptomatology.

In conclusion, this study demonstrates that both boys and girls with combined type ADHD have increased levels of PDD symptoms. Although the major classification systems (DSM-IV and ICD-10) discourage diagnosing a combination of ADHD and PDD, our results as well as others' speak against this strict segregation. Furthermore, 
we found the familiality of PDD symptoms to be largely independent of the familiality of ADHD. Of all PDD dimensions, stereotyped behavior showed the strongest familiality. Our results suggest that PDD familiality may be higher in ADHD affected girls than in boys, but our female sample was of limited size. PDD familiality appeared age dependent, which stresses the importance of taking age into account when interpreting genetic study results and comparing between such studies. Future research may try to elucidate the genetic loci involved in PDD symptoms in the context of ADHD in comparisons with genetic findings in PDD as such. The implications of the presence of PDD symptoms for the prognosis and treatment of children with ADHD need attention.

Acknowledgements The IMAGE-study was supported by National Institute of Health grant 1 R01 MH62873-01A1 to S.V. Faraone.

Open Access This article is distributed under the terms of the Creative Commons Attribution Noncommercial License which permits any noncommercial use, distribution, and reproduction in any medium, provided the original author(s) and source are credited.

\section{References}

American Psychiatric Association (1994). Diagnostic and statistical manual of mental disorders (4th ed.). Washington DC: Author.

Bakker, S. C., van der Meulen, E. M., Buitelaar, J. K., Sandkuijl, L. A., Pauls, D. L., Monsuur, A. J., et al. (2003). A whole-genome scan in 164 Dutch sib pairs with attention-deficit/hyperactivity disorder: suggestive evidence for linkage on chromosomes $7 p$ and 15q. American Journal of Human Genetics, 72, 1251-1260. doi:10.1086/375143.

Berument, S. K., Rutter, M., Lord, C., Pickles, A., \& Bailey, A. (1999). Autism screening questionnaire: diagnostic validity. The British Journal of Psychiatry, 175, 444-451.

Brookes, K., Xu, X., Chen, W., Zhou, K., Neale, B., Lowe, N., et al. (2006). The analysis of 51 genes in DSM-IV combined type attention deficit hyperactivity disorder: association signals in DRD4, DAT1 and 16 other genes. Molecular Psychiatry, 11, 934-953. doi:10.1038/sj.mp.4001869.

Buitelaar, J. K., van der Wees, M., Swaab-Barneveld, H., \& van der Gaag, R. J. (1999). Theory of mind and emotion-recognition functioning in autistic spectrum disorders and in psychiatric control and normal children. Development and Psychopathology, 11, 39-58. doi:10.1017/S0954579499001947.

Clark, T., Feehan, C., Tinline, C., \& Vostanis, P. (1999). Autistic symptoms in children with attention deficit-hyperactivity disorder. European Child \& Adolescent Psychiatry, 8, 50-55. doi:10.1007/s007870050083.

Cohen, J. (1988). Statistical power analysis for the behavioral sciences (2nd ed.). Hillsdale, NJ: Erlbaum.

Conners, K. (1996). Rating scales in ADHD. Durham, NC: Duke University Medical Center.

Constantino, J. N., Hudziak, J. J., \& Todd, R. D. (2003). Deficits in reciprocal social behavior in male twins: evidence for a genetically independent domain of psychopathology. Journal of the American Academy of Child and Adolescent Psychiatry, 42, 458-467.
Constantino, J. N., Lajonchere, C., Lutz, M., Gray, T., Abbacchi, A., McKenna, K., et al. (2006). Autistic social impairment in the siblings of children with pervasive developmental disorders. The American Journal of Psychiatry, 163, 294-296. doi:10.1176/ appi.ajp.163.2.294.

Constantino, J. N., \& Todd, R. D. (2003). Autistic traits in the general population: a twin study. Archives of General Psychiatry, 60, 524-530. doi:10.1001/archpsyc.60.5.524.

Dyck, M. J., Piek, J. P., Hay, D., Smith, L., \& Hallmayer, J. (2006). Are abilities abnormally interdependent in children with autism? Journal of Clinical Child and Adolescent Psychology, 35, 20-33. doi:10.1207/s15374424jccp3501 3.

Faraone, S. V., Perlis, R. H., Doyle, A. E., Smoller, J. W., Goralnick, J. J., Holmgren, M. A., et al. (2005). Molecular genetics of attention-deficit/hyperactivity disorder. Biological Psychiatry, 57, 1313-1323. doi:10.1016/j.biopsych.2004.11.024.

Farzin, F., Perry, H., Hessl, D., Loesch, D., Cohen, J., Bacalman, S., et al. (2006). Autism spectrum disorders and attention- deficit/ hyperactivity disorder in boys with the fragile $\mathrm{X}$ permutation. Developmental and Behavioural Pediatrics, 27, 137-144. doi:10.1097/00004703-200604002-00012.

Freitag, C. M. (2007). The genetics of autistic disorders and its clinical relevance: a review of the literature. Molecular Psychiatry, 12, 222. doi:10.1038/sj.mp.4001896.

Georgiades, S., Szatmari, P., Zwaigenbaum, L., Duku, E., Bryson, S., Roberts, W., et al. (2007). Structure of the autism symptom phenotype: A proposed multidimensional model. Journal of the American Academy of Child and Adolescent Psychiatry, 46, 188196. doi:10.1097/01.chi.0000242236.90763.7f.

Geurts, H. M., Luman, M., \& van Meel, C. S. (2008). What's in a game: the effect of social motivation on interference control in boys with ADHD and autism spectrum disorders. Journal of Child Psychology and Psychiatry, and Allied Disciplines, 49(8), 848-857.

Geurts, H. M., Verte, S., Oosterlaan, J., Roeyers, H., \& Sergeant, J. A. (2004). How specific are executive functioning deficits in attention deficit hyperactivity disorder and autism? Journal of Child Psychology and Psychiatry, and Allied Disciplines, 45, 836-854. doi:10.1111/j.1469-7610.2004.00276.x.

Goin-Kochel, R. P., Abbacchi, A., \& Constantino, J. N. (2007). Lack of evidence for increased genetic loading for autism among families of affected females: a replication from family history data in two large samples. Autism, 11, 279-286. doi:10.1177/ 1362361307076857.

Goin-Kochel, R. P., Mazefsky, C. A., \& Riley, B. P. (2008). Level of functioning in autism spectrum disorders: phenotypic congruence among affected siblings. Journal of Autism and Developmental Disorders, 38, 1019-1027. doi:10.1007/s10803-007-0476-z.

Goodman, R. (1997). The Strengths and Difficulties Questionnaire: a research note. Journal of Child Psychology and Psychiatry, and Allied Disciplines, 38, 581-586. doi:10.1111/j.1469-7610.1997. tb01545.x.

Groth-Marnat, G. (1997). Handbook of psychological assessment (3rd ed.). New York: Wiley.

Happé, F., Booth, R., Charlton, R., \& Hughes, C. (2006). Executive function deficits in autism spectrum disorders and attentiondeficit/hyperactivity disorder: examining profiles across domains and ages. Brain and Cognition, 61, 25-39. doi:10.1016/j. bandc.2006.03.004.

Hartman, C. A., Luteijn, E., Moorlag, H., de Bildt, A., \& Minderaa, R. B. (2007a). Vragenlijst voor Inventarisatie van Sociaal gedrag van Kinderen (VISK). Herziene handleiding 2007. Amsterdam: Harcourt Test Publishers.

Hartman, C. A., Luteijn, E., Moorlag, H., de Bildt, A., \& Minderaa, R. B. (2007b). The Children's Social Behavior Questionnaire (CSBQ). Revised manual 2007. The Netherlands: University Medical Center Groningen Unpublished manuscript. 
Hartman, C. A., Luteijn, E., Serra, M., \& Minderaa, R. (2006). Refinement of the children's social behavior questionnaire (CSBQ): an instrument that describes the diverse problems seen in milder forms of PDD. Journal of Autism and Developmental Disorders, 36, 325-342. doi:10.1007/s10803-005-0072-z.

Jonsdottir, S., Bouma, A., Sergeant, J. A., \& Scherder, E. J. (2006). Relationships between neuropsychological measures of executive function and behavioral measures of ADHD symptoms and comorbid behavior. Archives of Clinical Neuropsychology, 21, 383-394. doi:10.1016/j.acn.2006.05.003.

Knafo, A., \& Plomin, R. (2006). Prosocial behavior from early to middle childhood: genetic and environmental influences on stability and change. Developmental Psychology, 42, 771-786. doi:10.1037/0012-1649.42.5.771.

Kolevzon, A., Smith, C. J., Schmeidler, J., Buxbaum, J. D., \& Silverman, J. M. (2004). Familial symptom domains in monozygotic siblings with autism. American Journal of Medical Genetics Part B, 129, 76-81. doi:10.1002/ajmg.b.30011.

Krug, D. A., Arick, J. R., \& Almond, P. J. (1980). Autism screening instrument for educational planning. Portland: ASIEP Education.

Luteijn, E. F., Serra, M., Jackson, S., Steenhuis, M. P., Althaus, M., Volkmar, F., et al. (2000). How unspecified are disorders of children with a pervasive developmental disorder not otherwise specified? A study of social problems in children with PDD-NOS and ADHD. European Child \& Adolescent Psychiatry, 9, 168179. doi:10.1007/s007870070040.

Muhle, R., Trentacoste, S. V., \& Rapin, I. (2004). The genetics of autism. Pediatrics, 113, 472-486. doi:10.1542/peds.113.5.e472.

Mulligan, A., Anney, R. J., O'Regan, M., Chen, W., Butler, L., Fitzgerald, M., et al. (2008). Autism symptoms in attention-deficit/hyperactivity disorder: a familial trait which correlates with conduct, oppositional defiant, language and motor disorders. Journal of Autism and Developmental Disorders; epub ahead of print.

Nijmeijer, J. S., Minderaa, R. B., Buitelaar, J. K., Mulligan, A., Hartman, C. A., \& Hoekstra, P. J. (2008). Attention-deficit/ hyperactivity disorder and social dysfunctioning. Clinical Psychology Review, 28, 692-708. doi:10.1016/j.cpr.2007.10.003.

Nyden, A., Gillberg, C., Hjelmquist, E., \& Heiman, M. (1999). Executive function/attention deficits in boys with Asperger Syndrome, attention disorder and reading/writing disorder. Autism, 3, 213-228. doi:10.1177/1362361399003003002.

Ogdie, M. N., MacPhie, I. L., Minassian, S. L., Yang, M., Fisher, S. E., Francks, C., et al. (2003). A genomewide scan for attentiondeficit/hyperactivity disorder in an extended sample: suggestive linkage on 17p11. American Journal of Human Genetics, 72, 1268-1279. doi:10.1086/375139.

Ozonoff, S., \& Jensen, J. (1999). Brief report: specific executive function profiles in three neurodevelopmental disorders. Journal of Autism and Developmental Disorders, 29, 171-177. doi:10.1023/A:1023052913110.

Preacher, K. J. (2002). Calculation for the test of the difference between two independent correlation coefficients [Computer software]. Available from http://www.quantpsy.org.

Raskind, W. H., Hsu, L., Berninger, V. W., Thomson, J. B., \& Wijsman, E. M. (2000). Familial aggregation of dyslexia phenotypes. Behavior Genetics, 30, 385-396. doi:10.1023/A:1002700605187.

Reiersen, A. M., Constantino, J. N., Volk, H. E., \& Todd, R. D. (2007). Autistic traits in a population-based ADHD twin sample. Journal of Child Psychology and Psychiatry, and Allied Disciplines, 48, 464472. doi:10.1111/j.1469-7610.2006.01720.x.
Ronald, A., Happé, F., Bolton, P., Butcher, L. M., Price, T. S., Wheelwright, S., et al. (2006a). Genetic heterogeneity between the three components of the autism spectrum: a twin study. Journal of the American Academy of Child and Adolescent Psychiatry, 45, 691-699. doi:10.1097/01.chi.0000215325. 13058.9d.

Ronald, A., Happé, F., \& Plomin, R. (2005). The genetic relationship between individual differences in social and nonsocial behaviours characteristic of autism. Developmental Science, 8, 444-458. doi:10.1111/j.1467-7687.2005.00433.x.

Ronald, A., Happé, F., Price, T. S., Baron-Cohen, S., \& Plomin, R. (2006b). Phenotypic and genetic overlap between autistic traits at the extremes of the general population. Journal of the American Academy of Child and Adolescent Psychiatry, 45, 1206-1214. doi:10.1097/01.chi.0000230165.54117.41.

Ronald, A., Simonoff, E., Kuntsi, J., Asherson, P., \& Plomin, R. (2008). Evidence for overlapping genetic influences on autistic and ADHD behaviours in a community twin sample. Journal of Child Psychology and Psychiatry, and Allied Disciplines, 49, 535-542. doi:10.1111/j.1469-7610.2007.01857.x.

Rutter, M., Anderson-Wood, L., Beckett, C., Bredenkamp, D., Castle, J., Groothues, C., et al. (1999). Quasi-autism patterns following severe early global deprivation. Journal of Child Psychology and Psychiatry, and Allied Disciplines, 40, 537-549. doi:10.1017/ S0021963099003935.

Santosh, P. J., \& Mijovic, A. (2004). Social impairment in hyperkinetic disorder-relationship to psychopathology and environmental stressors. European Child \& Adolescent Psychiatry, 13, 141-150. doi:10.1007/s00787-004-0372-4.

Scourfield, J., John, B., Martin, N., \& McGuffin, P. (2004). The development of prosocial behaviour in children and adolescents: a twin study. Journal of Child Psychology and Psychiatry, and Allied Disciplines, 45, 927-935. doi:10.1111/j.1469-7610.2004. t01-1-00286.x.

Smalley, S. L., Kustanovich, V., Minassian, S. L., Stone, J. L., Ogdie, M. N., McGough, J. J., et al. (2002). Genetic linkage of attentiondeficit/hyperactivity disorder on chromosome 16p13, in a region implicated in autism. American Journal of Human Genetics, 71, 959-963. doi:10.1086/342732.

Szatmari, P., Merette, C., Emond, C., Zwaigenbaum, L., Jones, M. B., Maziade, M., et al. (2008). Decomposing the autism phenotype into familial dimensions. American Journal of Medical Genetics Part B, 22, 3-9. doi:10.1002/ajmg.b.30561.

Taylor, E. A. (1986). Childhood hyperactivity. British Journal of Psychiatry, 149, 562-573.

Wechsler, D. (2000). WAIS-III Nederlandstalige bewerking. Technische handleiding. London: The Psychological Corporation.

Wechsler, D. (2002). WISC-III Handleiding. London: The Psychological Corporation.

World Health Organisation (1992). International Classification of Diseases -10th revision. Geneva: Author.

Yamagata, T., Aradhya, S., Mori, M., Inoue, K., Momoi, M. Y., \& Nelson, D. L. (2002). The human secretin gene: fine structure in $11 \mathrm{p} 15.5$ and sequence variation in patients with autism. Genomics, 80, 185-194. doi:10.1006/geno.2002.6814.

Yuill, N., \& Lyon, J. (2007). Selective difficulty in recognising facial expressions of emotion in boys with ADHD : General performance impairments or specific problems in social cognition? European Child \& Adolescent Psychiatry, 16, 398-404. doi:10.1007/s00787-007-0612-5. 